\section{Mass extinctions and missing matter}

SIR - The approximate 30-Myr periodicity that appears to exist in the record of mass biological extinctions and terrestrial impact cratering has been interpreted by Rampino and myself ${ }^{1}$ as evidence of a quasi-periodic bombardment of the Earth by comets that have been perturbed into Earth-crossing orbits by close gravitational encounters of the Solar System with massive interstellar clouds of gas and dust. In this model, the underlying clockwork is the Solar System's vertical oscillation through the midplane of the Galaxy, towards which most of the massive interstellar clouds are concentrated.

The downward force exerted by the Galaxy will also perturb the Solar System's comet halo, but will not produce any periodicity. To see why, first observe that the Solar System's vertical orbit ${ }^{2}$ lies wholly within the linear domain of the galactic force law ${ }^{3,4}$, so that the vertical galactic acceleration is simply $K(z)=$ - $4 \pi G \varrho_{0} z$, where $\varrho_{0}$ represents the local mass density of material near the galactic plane. It follows that the difference between the galactic acceleration of the Sun at galactic height $z$ and that of a comet at a distance $r$ from the Sun in the direction of the galactic plane is $K(z)-K(z-r)=$ $-4 \pi G \varrho_{0} r$. By equating this small galactic tidal acceleration to the local acceleration of the comet towards the Sun, $-G M_{\odot} / r^{2}$, a 'tidal radius' for the comet halo is readily obtained: $r_{t}=\left(M_{\odot} / 4 \pi \varrho_{0}\right)^{1 / 3}=1.5 \times$ $10^{5} \mathrm{AU}$. (For comparison, the Galaxy's central force field and differential rotation impose a tidal limit ${ }^{5}$ of $3 \times 10^{5} \mathrm{AU}$.) Because of its independence of galactic height, $r_{t}$ differs from the tidal radius that is derived for an inverse-square galactic force law, valid very far from the galactic plane ${ }^{6}$. Thus, the comet halo, unlike a farranging globular star cluster ${ }^{7}$, never experiences a periodic 'gravitational shock' as it passes up and down through the galactic disk (contrary to ref. 8), although it does suffer a constant amount of compression in the vertical direction. For this reason, individual objects like interstellar gas and dust clouds seem to be needed as perturbers.

Could other dark perturbing objects also be important? The disk of our Galaxy probably contains a large quantity of 'invisible matter'. Kinematical studies have produced a range of estimates for the total mass density $e_{0}$ in the solar neighbourhood (believed to be typical of the part of the galactic plane that is accessible to the Solar System) equal to $0.14-0.24 M_{\odot} \mathrm{pc}^{-3}$ (refs $3,4,9-12$ ). Direct counts of observed stars, gas, and dust have turned up only 0.10 $0.11 M_{\odot} \mathrm{pc}^{-3}$ (refs 4,10 ), and so the missing disk material could amount to as much as $\sim 0.14 \mathrm{M}_{\odot} \mathrm{pc}^{-3}$.

The effectiveness of any seen or unseen matter in perturbing the Solar System's comet halo depends only on the local mass density of this matter, $e_{0}$. From equations (11) and (12) of Hills ${ }^{13}$, the mean time interval between random encounters of the Solar System with objects of mass density $\varrho$ is given by $\tau_{\mathrm{s}}=\varrho^{-1} a^{-2}{ }_{\min } \mathrm{Myr}$, if $\varrho$ is in units of $M_{\odot} \mathrm{pc}^{-3}$ and $a_{\min }$ in units of $2 \times$ $10^{4} \mathrm{AU}$, where $a_{\min }$ is the minimum semimajor axis of those comets for which the loss cone is filled by an encounter. (This result depends on the impulse approximation and point-mass approximation, which turn out to be adequate assumptions for all cases considered here.) Hills' equations (13) and (19) (ref.13) (with $n=$ 2) provide the total number of comet collisions with the Earth during an encounter, $N=N_{0} a^{-2}$ min, where $N_{0}=4$ or $N_{0}=0.2$ depending on whether Everhart's ${ }^{14}$ or Weissman's ${ }^{15}$ rate of present-day comet impacts is assumed. In either case, the shower of comets ${ }^{13}$ lasts $\tau_{1}$ $=11 a^{3 / 2}{ }_{\min }$ Myr. Because the outer radius of the main comet reservoir probably lies at $\sim 2 \times 10^{4} \mathrm{AU}$ (refs 13,16-19), $a_{\min }$ is not likely to exceed 1 .

For the Solar System's vertical motion within the Galaxy to significantly modulate the background comet flux arising from random showers, the most important perturbing objects must not only have a high mass density but must also be concentrated fairly strongly towards the galactic plane. The total comet flux into the inner Solar System is due to solar encounters with known stars $\left(\sim 0.05 M_{\odot}\right.$ $\mathrm{pc}^{-3}$ ) (ref.4), known interstellar gas and dust clouds $\left(\sim 0.05 M_{\odot} \quad \mathrm{pc}^{-3}\right.$ (ref.20), interstellar comets $\left(<6 \times 10^{-4} M_{\odot} \mathrm{pc}^{-3}\right.$ (ref.21), and invisible matter $\left(\leq 0.14 M_{\odot}\right.$ $\mathrm{pc}^{-3}$ ). If the invisible matter consists of small (or cold) interstellar molecular clouds, which would probably be concentrated strongly toward the galactic plane like the known molecular clouds and the youngest stars $\left(\sim 0.04 M_{\odot} \mathrm{pc}^{-3}\right.$ ) (refs 4,20 ), then the total percentage of local material lying in the flat subsystem could be as high as $\mathbf{7 5 \%}$. Even without invisible matter, the total percentage would still be fairly high, $40 \%$. In all cases, the encounter time $\tau_{\text {s }}$ would be acceptably shorter than the Solar System's galactovertical halfperiod, $P_{1 / 2}=\left(\pi / 4 G Q_{0}^{\wedge}\right)^{1 / 2} \approx 30 \mathrm{Myr}$, and so galactic modulation could arise.

If, however, the invisible material consists of a large population of old, dead stars, with a relatively small galactic concentration, the percentage of local matter belonging to the flat subsystem could be as low as $17 \%$. This would be insufficient to modulate the background comet flux into the inner Solar System. It might be argued that most of these dead stars are young objects, not yet kinematically dispersed far from the galactic plane. However, they would also have to be old enough to have migrated into the area between the starforming spiral arms (so as to adequately cover the region of the galactic plane that the Solar System traverses in its revolution around the galactic centre). Their ages would consequently ${ }^{22}$ need to lie in the narrow range $10^{8}-10^{9} \mathrm{yr}$. Yet it is very unlikely that much of the invisible matter consists of dead stars; the space densities of faint white dwarfs ${ }^{23}$ and of black dwarfs ${ }^{24-}$ ${ }^{26}$ are observed to fall off rapidly with declining luminosity (at least to $M_{\mathrm{v}}=21$ ), while most neutron stars and stellar black holes are expected to be derivatives of massive main-sequence stars, whose space densities, at least today, are observed to be extremely low ${ }^{4}$.

If future observations reveal a large secondary maximum in the luminosity functioin of dead stars at a lower light level (perhaps because star formation has been very sporadic), the implications for the present hypothesis of Galaxy-modulated comet impacts on Earth ${ }^{1}$ could be profound.

\section{National Aeronautics and Space}

Administration,

Goddard Space Flight Center,

Institute for Space Studies,

New York, New York 10025, USA

1. Rampino, M.R. \& Stothers, R.B. Nature 308, 709-712 (1984).

2. Innanen, K.A., Patrick, A.T. \& Duley, W.W. Astrophys. Space Sci. 57, 511-515 (1978).

3. Oort, J.H. in Galactic Structure (eds Blaauw, A. \& Schmidt, M.) 455-511 (University of Chicago, 1965).

4. Bahcall, J.N. Astrophys. J. 276, 169-181 (1984)

5. Wyatt, S.P. \& Faintich, M.B. Bull. Am. astr. Soc. 3, 368 (1971).

6. von Hoerner, S. Astrophys. J. 125, 451-469 (1957).

7. Ostriker, J.P., Spitzer, L. Jr \& Chevalier, R.A. Astrophys. J. 176, L51-56 (1972).

8. Scaio, J.M. \& Smoluchowski, R. Bull. Am. astr. Soc. 16, 493-494 (1984).

9. Krisciunas, K. Astrophys. J. 82, $195-197$ (1977)

10. Hill, G., Hilditch, R.W. \& Barnes, J.V. Mon. Not. R. astr. Soc. 186, 813-830 (1979).

11. Rohlfs, K. \& Kreitschmann, J. Astrophys. Space Sci. 79. 289.319 (1981).

12. Eggen, O.J. Publs. astr. Soc. Pacif. 81, $741-753$ (1969).

13. Hills, J. G. Astrophys. J. 86, 1730-1740 (1981).

14. Everhart, E. Astrophys. J. 74, 735-750 (1969).

15. Weissman, P.R. Astr. Astrophys. 85, 191-196 (1980).

16. Fernandez, J.A. Icarus 42, 406-421 (1980).

17. Napier, W.M. \& Staniucha, M. Mon. Not. R. astr. Soc. 198, 723-735 (1982).

18. van den Bergh, S. J. R. astr. Soc. Can. 76, 303-308 (1982). 19. Bailey, M.E. Mon. Not. R. astr. Soc. 204, 603-633 (1983). 20. Sanders, D. B., Solomon, P.M. \& Scoville, N.Z. Astrophys. J. 276, 182-203 (1984).

21. Sekanina, Z. Icarus 27, 123-133 (1976).

22. Wielen, R. Astr. Astrophys. 60, 263-275 (1977).

23. Liebert, J., Dahn, C.C., Gresham, M. \& Strittmatter, P.A Astrophys. J. 233, 226-238 (1979).

24. Probst, R.G. \& O'Connell, R.W. Astrophys. J. Lett.252, L69-L72 (1982)

25. Jameson, R.F., Sherrington, M.R. \& Giles, A.B. Mon. Not, R. astr. Soc. 205, 39P-41P (1983)

26. Reid, N. Mon. Not. R. astr. Soc. 206, 1-17 (1984)

\section{The case against impact extinctions}

SIR - Some people think that the impact hypothesis for the mass extinction at the Cretaceous-Paleogene boundary is 'proved'1,2. So it is, if one considers selectively one set of evidence. However, it is equally convincingly disproved if one considers selectively another set of evidence. I have recently reviewed this topic ${ }^{3}$, evaluating the literature through 1983; no valid later work known to me materially affects any of the many arugements in each direction 